\title{
Patient and public involvement in the
} design, administration and evaluation of patient feedback tools, an example in psychiatry: a systematic review and critical interpretative synthesis.

Baines, Rebecca

http://hdl.handle.net/10026.1/12915

10.1177/1355819618811866

Journal of Health Services Research and Policy

SAGE Publications

All content in PEARL is protected by copyright law. Author manuscripts are made available in accordance with publisher policies. Please cite only the published version using the details provided on the item record or document. In the absence of an open licence (e.g. Creative Commons), permissions for further reuse of content should be sought from the publisher or author. 


\section{Background:}

Patient feedback is considered integral to healthcare delivery, design and reform. Although there is a strong policy commitment to evidencing patient and public involvement (PPI) in the design of patient feedback tools, it is not clear that this happens in practice.

\section{Methods:}

A systematic review using thematic analysis and critical interpretative synthesis of peerreviewed and grey literature published between 2007-2017 exploring the presence of PPI in the design, administration and evaluation of patient feedback tools for practising psychiatrists. Research process was done in collaboration with a volunteer mental health patient research partner.

\section{Results:}

Fourteen (10 peer-reviewed, four grey literature) articles discussing the development of nine patient feedback tools were included. Six of the nine tools reviewed were designed from a professional perspective only. Criteria generation and categorisation also primarily remained at the professional's discretion. Patient participation rates, presence of missing data, and psychometric validation were used to determine validity and patient acceptability. In most instances, patients remained passive recipients with limited opportunity to actively influence change at any stage. No article reviewed reported PPI in all aspects of tool design, administration or evaluation.

\section{Conclusions:}

The majority of patient feedback tools are designed, administered and evaluated from the professional perspective only. Existing tools appear to assume that: professional and patient agendas are synonymous; psychometric validation is indicative of patient acceptability; and psychiatric patients do not have the capacity or desire to be involved. Future patient feedback tools in any healthcare setting should be co-produced from the outset to ensure they are valued by all those involved. A reconsideration of the purpose of patient feedback and what constitutes valid patient feedback is also required. 


\section{Introduction}

Patient feedback is considered integral to professional development, healthcare service design, delivery, and reform..$^{1-4}$ However, despite extensive policy requirements, recent research challenges the perceived efficacy, validity and acceptability of existing patient feedback tools. ${ }^{56}$ While policy driven discourses promote patient and public involvement (PPI) in the design, administration and evaluation of such tools, ${ }^{7}$ critical exploration of this in practice is severely limited, particularly within the field of psychiatry. ${ }^{8-10}$

Typically collected through validated questionnaires or surveys, ${ }^{11} 12$ some doctors and patients remain sceptical about the reliability of existing patient feedback tools due to: the adoption of generic questionnaires that exclude vulnerable patient groups; the inability of existing tools to ensure sufficient patient representation; biased patient selection i.e. choosing the "right" patient to participate, and biased patient responses, i.e. patients not providing honest feedback due to concerns for future healthcare delivery. ${ }^{6}$ Sir Keith Pearson, the lead for a recent independent review of medical regulation in the UK expressed some doubt that existing questionnaires "provide sufficient quality and breadth of information to enable a doctor to reflect properly on their interaction with patients..." ${ }^{5}$ The practicality and acceptability of existing patient feedback tools is therefore contested from both a patient and professional perspective inhibiting their adoption and subsequent benefit to both patients and the profession. ${ }^{56}$

Furthermore, in addition to the generic issues associated with patient feedback tools discussed above, psychiatrists experience specific concerns. For example, psychiatrists frequently express concerns about a patient's ability to "know their own mind", 1314 reflecting issues of capacity. In some instances, patients who 'lack capacity' are excluded from patient feedback opportunities. While substantial improvements have been made with regard to shared decision making processes,${ }^{13}$ evidence suggests mental health patients are frequently excluded, or at best under-represented in experiencing patient feedback opportunities. ${ }^{15}$

Such findings are concerning given the valuable learning opportunities attributed to patient feedback. ${ }^{16} 17$ Pertinent to the context of this review, some authors argue that exploring patient feedback in mental health is of paramount importance as patients are more likely to 
maintain contact with medical services if they are satisfied with their care. ${ }^{18}$ This in turn, has implications for reducing clinical relapse incidents and hospital admission rates affecting patient wellbeing and resource expenditure. ${ }^{18}$ It is worth noting, contact periods for mental health services are typically longer than those related to physical conditions. Understanding and acknowledging the patient perspective in the design, administration and evaluation of patient feedback tools is therefore imperative as the continued use of ineffective methods has been shown to be detrimental to the overall quality of care received. ${ }^{19}$ Despite this, critical exploration of PPI in the design of patient feedback tools is limited, particularly within the field of psychiatry. ${ }^{8-10}$

This review therefore aims to critically examine: how, if at all, patients and the public are involved in the design, administration and evaluation of patient feedback tools for practising psychiatrists. By identifying potential flaws, assumptions and contradictions (the central aim of critical interpretative synthesis) of existing tools, practical and policy related implications can be identified. Although psychiatry is used as an example for the purposes of this review, its findings and conclusions are likely to have valuable lessons for the use of PPI in the design of patient feedback tools in healthcare more generally.

For the purposes of this review, the term patient is used to be inclusive of service-users, consumers, clients, carers and family members although the important distinctions between these terms are acknowledged.

\section{Methods}

This systematic review followed the Preferred Reporting Items for Systematic Reviews and Meta-Analysis (PRISMA) flow diagram ${ }^{20}$ and guidance set out by the Centre for Reviews and Dissemination. ${ }^{21}$ The review's protocol is available on the PROSPERO register registration number CRD42016050533.

All research was conducted in collaboration with a volunteer mental health patient research partner who has extensive experience of receiving psychiatric care. Published principles of PPI were followed to support this involvement. ${ }^{22}$ 


\section{Search strategy}

\section{Peer-reviewed literature}

Search terms listed in Table 1 were designed and reviewed using the Peer Review of Electronic Search Strategies (PRESS) guidance ${ }^{23}$. A scoping exercise revealed the need to produce an extensive list of patient synonyms to remain sensitive to different contexts (Table 1). As advised by an information specialist, agreed search terms were used to systematically search: MEDLINE; PubMed; PsycINFO; Embase, CINAHL and Cochrane Library databases for articles published in the English language. Database searches were supplemented by reference list searches of included studies. Two independent reviewers conducted the research process.

\section{Grey literature}

Grey literature, defined as 'that which is produced on all levels of government, academics, business and industry in print and electronic formats, but which is not controlled by commercial publishers, ${ }^{24}$ was also searched using Google to ensure sufficient coverage. Screening was limited to the first 10 pages in order to maintain a manageable sample size. The peer-reviewed search strategy was also used to search Google Scholar.

Table 1 Search term strategy

\section{Inclusion criteria}

Articles were reviewed independently by two authors using a two stage process. Firstly, all identified articles were screened by their title and abstracts using a pre-defined inclusion criteria form (Table 2). Rayyan, a web application for systematic reviews was used to facilitate this process. ${ }^{25}$ Where a decision could not be made, the full article was retrieved. Full texts of included articles were then reviewed for inclusion. Discrepancies would have been resolved with reference to a third reviewer. This process was not required.

To develop a manageable focus, studies: not in the English Language, published prior to 2007; with a specialty specific focus of child, Dementia/Alzheimer's or learning difficulties were excluded as these areas are likely to require tailored patient feedback activities beyond the remit of this review. Any study design except opinion, commentary or letter articles were included. The date parameters of 2007-2017 were selected to ensure only contemporary information was included. Similarly, only English language studies were included as the authors could not ensure an accurate translation. 
For clarity, articles that described the evaluation or assessment of mental health services but did not differentiate individual psychiatrist involvement were excluded. Inclusion decisions are documented in Figure 1.

Table 2 Inclusion criteria form

\section{Data analysis and synthesis}

Data were thematically analysed using an inductive approach and synthesised using critical interpretative synthesis (CIS). ${ }^{26} 27$ Two researchers were involved in the coding and analysis of results. Relevant text under the headings results, discussion and/or conclusions were extracted electronically with thematic analysis supported through the use Nvivo 11 software. ${ }^{28}$ Two researchers initially reviewed two papers line by line to develop a comprehensive coding framework. The framework was then used to individually analyse all included studies and to iteratively compare emerging themes across studies to determine dominant themes. Themes were then synthesised using CIS.

$\mathrm{CIS}$ is an adaptation of meta-ethnography and borrows techniques from Grounded Theory. ${ }^{29}$ CIS was chosen as the synthesis methodology for this review due to its ability to integrate both qualitative and quantitative evidence enabling the generation of theory. ${ }^{30}$ It incorporates conventional systematic review methodology with traditional techniques of qualitative enquiry enabling the aggregation and examination of existing literature as a corpus of information, and generation of new perspectives through the critical interrogation of existing contradictions, flaws and assumptions between, not within individual studies. Essentially, CIS seeks to problematise the literature. One of its defining features is its ability to generate synthetic constructs, a "third order construct", the result of transforming underlying evidence into new conceptual forms, and synthesising argument(s). ${ }^{27}$ As Dixonwoods explains: "this argument integrates evidence... into a coherent theoretical framework... to provide more insightful, formalised, and generalisable ways of understanding a phenomenon". ${ }^{27}$ The CIS stages set out by Flemming (2010) were followed. ${ }^{30}$ The flaws, assumptions and contradictions identified through this review are used to structure its discussion.

\section{Quality assessment}

Buckley et al's criteria ${ }^{31}$ were used to quality appraise included peer-reviewed studies. In line with previous research, ${ }^{32}$ studies scoring seven or above were regarded as high quality. Finally, 
sensitivity analyses, a form of analysis that tests for the effect on conclusions drawn when lower quality studies are excluded, were also conducted. Such analyses are considered an important focus of any qualitative synthesis method warranting their inclusion. ${ }^{33}$ Consistent with the synthesis method chosen, conceptual relevance took preference over methodological rigour. ${ }^{27}$ Quality appraisal of included studies were conducted independently by two reviewers until consensus was achieved.

Figure 1 PRISMA peer review inclusion process

Figure 2 PRISMA grey literature inclusion process

\section{Results}

\section{Study characteristics}

\section{Peer-reviewed literature}

Database and reference list searches of included articles led to the total inclusion of ten peer-reviewed studies discussing nine unique patient feedback tools (Figure 1). Studies were published primarily in the UK $(n=5)$ with others published in Canada $(n=1)$; Sweden $(n=1)$; the US ( $n=1)$; Italy $(n=1)$; and France $(n=1)$. Included study characteristics are outlined in Table 3.

\section{Grey literature}

Grey literature searches identified 107 unique documents leading to the total inclusion of four relevant documents (Figure 2). ${ }^{734-36}$ These are presented alongside the peer-review findings.

Fourteen (10 peer-reviewed, four grey literature) articles discussing the development of nine patient feedback tools were therefore included. Included study characteristics are listed in Table 3.

\section{Study quality}

Six peer-reviewed articles were appraised as high quality (Table 3).. Sensitivity analyses indicated that the exclusion of studies assessed as low quality (i.e. appraisal score of below 
seven), did not affect the conclusions drawn. All studies were therefore retained to be inclusive of the available literature.

Table 3 Included study characteristics

Results are presented in order of some of the generic steps involved in tool development:

- Step 1: Tool Design, e.g. whose perspective will the tool be designed from? What information will the content of the tool be designed from? Which domains and questions will the tool cover? How will the questions be phrased? And how will respondents answer the questions planned, what response methods will they use?

- Step 2: Tool administration, e.g. who will administer the tool? How?

- Step 3: Tool evaluation, e.g. piloting and validation.

\section{Patient and Public Involvement in patient feedback tool design}

Design perspective

Six of the nine tools reviewed were designed from a professional perspective only. ${ }^{74-42}$ For example, the ACP 360, a tool used by the Royal College of Psychiatrists in the UK described how it "is the only tool designed and validated for psychiatrists, by psychiatrists". ${ }^{36}$ One article reviewed did not discuss the design perspective and was assumed to be from that of the professional. ${ }^{43}$ Professional perspectives were often obtained through professional steering or working groups, and pre-defined authoritative guidelines, such as Good Medical Practice.$^{40}$ One tool was designed from a combination of the patient and professional perspective. ${ }^{8}$ Two tools reviewed were also designed from the patient perspective with minimal professional input (Table 4). ${ }^{44} 45$

\section{Tool content}

Where discussed, the generation of tool criteria was dominated by professional input $(n=3 / 5) .{ }^{374042}$ Authors of one article reported only criteria "the regulatory authority and the physicians themselves believed to be important" (p.529) were included. ${ }^{37}$ In most instances, professional opinion and authoritative guidelines were taken as a proxy measure for the patient voice and their desires.

"However, in two cases tool content was not driven by the profession alone. ${ }^{44} 45$ Following interviews with 20 patients of whom 17 had in-patient psychiatric care experience, five descriptive categories of care quality were developed: patient dignity and respect; a patient's sense of security e.g. trust; patient participation in care; patient recovery e.g. 
supportive guidance, opportunities for post-care follow-up; and a patient's care environment e.g. personal space and aesthetics. ${ }^{44}$ Another study used face-to-face semistructured interviews with 80 in-patients with various diagnoses including, schizophrenia, bipolar, mental and behavioural disorders due to psychoactive substance use. ${ }^{45}$ Patient interview data was then used to determine question design and response scales used, additional aspects of tool design also discussed in this review..

However, while often described as patient generated, the categorisation or analysis of suggested content was ultimately at the professionals' discretion. ${ }^{42} 45$ No article reviewed discussed the analysis of data in collaboration with patients or members of the public. A tool's content was a further area in which professional opinion typically superseded patient contributions.

Finally, the number of domains covered in patient feedback tools varied from four ${ }^{43}$ to eight. ${ }^{45}$ The number of questions used to assess these domains also varied ( $\left.n=5-69\right)$. No papers reported patient involvement in discussions around the number of domains included or number of questions asked. No clear rationale behind the variability of included item numbers and assessment domains was identified.

\section{Question design}

PPI in the formatting of proposed questions was mixed. Where drawn upon, patient involvement was favourably described. ${ }^{8}$ For example, Barbato et al. acknowledged how participating patients prompted the importance of a more direct and friendly style of questioning e.g. 'do you get on well with your psychiatrist?' ${ }^{8}$ When not involved, authors reported high "unable to rate" responses ${ }^{42}$ and patient response confusion. ${ }^{40}$ For example, despite being tested for "face validity and feasibility by eight consultants and their colleagues only...." one acknowledged limitations of Lelliot et al.'s tool was the "substantial number of patients unable to rate some items. ${ }^{\prime 42}$ Campbell et al. also identified one item in particular that caused some patient confusion. ${ }^{40}$ When responding to the statement "I have no reservation about seeing this doctor again", 87 respondents altered their initial binary response i.e. yes or no, following a misunderstanding or misreading of the question ${ }^{40}$ This statement (related to reservation) also had a substantially higher proportion of adverse ratings in comparison to other questions asked. ${ }^{40}$ 


\section{Response scales}

Response scales were defined by patients in two cases. ${ }^{845}$ Following patient suggestions, Barbato et al. used smileys in conjunction with a five point Likert scale (very positive-very negative),${ }^{8}$ and Zendjidjian adopted the language used by patients in preceding interviews as the response scale modalities for a five point Likert scale i.e. extremely less than expected - better than expected to facilitate patient understanding. ${ }^{45}$ No other articles discussed the decision process of response scale agreement in collaboration with patients or members of the public.

\section{Patient and public involvement in patient feedback tool administration} One article directly involved patients in the tool's delivery. ${ }^{8}$ Barbato et al. employed six patients to deliver the questionnaire, offer assistance if required, and to collect completed tools. Professional involvement in this process was deliberately kept to a minimum to limit possible conformity and overrating of positive answers, i.e. social desirability bias. ${ }^{8}$ The low refusal rate of $12 \%$ and wide patient representation achieved was directly attributed to PPI by the article's authors. ${ }^{8}$

\section{Patient and public involvement in patient feedback tool evaluation}

\section{Piloting}

Four articles reported the involvement of patients in the piloting of proposed tools. ${ }^{38} 394445$ In one instance, six patients recruited through a local patients' association with experience of psychiatric care were asked to complete the proposed questionnaire at home, and then evaluate it using a piloted checklist. ${ }^{44}$ Participating patients were required to assess each tool item in terms of: importance in care quality (1=very important, $5=$ of little importance); clarity e.g. clear and easy to understand, acceptable or unclear and hard to understand; general structure, relevance and usefulness. ${ }^{44}$ Participant evaluations were then discussed with researchers either face-to-face or by phone, leading to the exclusion of 58 items due to perceived importance $(n=10)$; emotionally charged or overlapping content $(n=20)$, and small levels of perceived importance $(n=28)$. Two other included articles reported the reduction of question items, and re-wording of questions to facilitate understanding, following patient involvement in the piloting stage. ${ }^{38} 39$

However, in most cases, the piloting of tools was assessed by the profession with "their patients" (p.157) $)^{42}$ as passive recipients. For example, tool appropriateness was assessed by 
allowing "every physician to be assessed to review the questionnaires and provide feedback that was incorporated into the final" version. (p.256) ${ }^{37}$ Two reviewed tools reported the use of informal feedback by participating doctors only .4038

\section{Validation}

With the exception of one tool not yet validated, ${ }^{39}$ the psychometric properties of a patient feedback tool were often used to determine all aspects of validity and acceptability. For example, as stated by Campbell et al. "analysis of psychometric properties showed that both surveys were acceptable to patients and colleagues."(p.192) ${ }^{64}$ Tool acceptability was also assessed through the examination of patient participation and levels of missing data $(n=5 / 8)$ 374042 45: "The high rate of return from patients suggests that raters did not find the questionnaire over-burdensome"(p.159). ${ }^{42}$ Patient participation or response omission was therefore used as a default measure of tool acceptability regardless of the questions which were ignored or responses provided.

Finally, although large numbers of patients participated in the validation of proposed tools, ${ }^{40}$ validation was often passive with limited opportunities to influence change. In some instances, physicians selected their own patients to be involved in the tool's evaluation introducing possible bias. ${ }^{42}$

\section{Discussion}

This review addressed limitations of existing research by exploring the presence of PPI in the design, administration and evaluation of patient feedback tools for psychiatrists. The discussion below presents potential flaws, assumptions and contradictions in the argumentation or reasoning underpinning existing tool design, administration and evaluation leading to the generation of a synthesising argument that contributes to policy debates in healthcare more broadly and highlights a number of solutions to consider.

\section{Identified flaws}

Despite a strong policy commitment, ${ }^{7}$ existing patient feedback tools primarily rely on professional opinion. Professional discretion often supersedes patient input, or remains restricted with the ability to influence change severely limited. While this may reflect a need for 'scientific or statistical' assurances as later discussed, it may also reflect a power imbalance between patients and the profession and the traditional approach to keep these 
two communities distinct. ${ }^{46} \mathrm{~A}$ lack of consistent PPI across the tool development stages may also reflect acknowledged difficulties in effective PPI. ${ }^{22}$ However, difficulties encountered should not be used as a rhetoric to deter PPI efforts.

\section{Existing assumptions and contradictions}

Existing patient feedback tools are also potentially undermined by three assumptions. Firstly, tool developers assume that professional and patient agendas are aligned. Three included studies demonstrate that this is not the case. ${ }^{8447} \mathrm{~A}$ lack of concordance between patients and psychiatrists has been reported, as has a lack of commonality between carer, patient and psychiatrist experiences. ${ }^{8}$ Lelliot et al. acknowledged that colleagues i.e. professionals, and patients are two independent groups whose perceptions and experiences of consultants is derived from different perspectives..$^{42}$ It is therefore imperative, that those responsible for designing existing patient feedback tools acknowledge the disparity between these populations and do not take one to be representative of the other i.e. colleague interests to be indicative of patient desires, or vice versa. Secondly, those responsible for evaluating patient feedback tools assume psychometric validation, patient participation, or absence of missing data is indicative of patient acceptability. The authors argue that to determine patient acceptability from these measures alone is reductionist and jeopardises tool validity. ${ }^{44}$ For example, if patients perceive the content or process of the tool to be irrelevant, they are unlikely to be motivated to complete it or provide accurate results, undermining the tools content and response process validity. As stated by Downing et al., ${ }^{48}$ once one validity domain is undermined, the ability of that tool to be used as an equitable assessment is also compromised. While important, the current reliance on psychometric validation alone is therefore unfavourable.

Finally, those looking to receive patient feedback sometimes assume that psychiatric patients do not have the capacity or desire to be involved. This assumption is directly challenged by a number of articles reviewed. ${ }^{8444547}$ An alternative interpretation of the reportedly low levels of patient engagement and assumed capacity is that it is the tool, and the traditionally exclusive approach to its design, administration and evaluation that lacks the capacity to facilitate effective patient involvement, not the participating individual. For example, while patients may not have the required capacity at a given point in time, this is 
unlikely be true for the entirety of their patient journey. The opportunity to provide patient feedback should therefore be patient dependent not physician dependent.

\section{Potential solutions}

We propose three solutions to address the identified flaws, assumptions and contradictions discussed. Firstly, co-producing tools from the outset is imperative. By its nature, coproducing tools may help to address acknowledged power imbalances and the underpinning assumptions that currently exist. Secondly, clarification of the intended purpose of patient feedback whether that be summative i.e. a point in time evaluation of performance against existing standards or benchmark, or formative i.e. an ongoing monitoring of performance, is required, as is its intended focus. ${ }^{40}$ Failure to do so is likely to create unnecessary confusion and development of poorly planned tools. An agreement of whether patient feedback tools seek to measure patient satisfaction, expectations or experience is also required as current ambiguity surrounding this issue complicates their development, administration and subsequent acceptance. Although often used interchangeably, each term represents discrete concepts that require different questions to be asked. As described by Gillet al. patient experience explores the specific experience of individuals, whilst patient satisfaction evaluates those individual experiences. ${ }^{49}$ In contrast, patient expectation is a measure of how well a patient's experience met their individual, and at times, highly variable expectations. If it is patient experience that regulators, employers or healthcare organisations seek to explore, then the overriding emphasis placed on psychometric validation must be reviewed. While it is important to validate proposed tools psychometrically if they are to be used as a summative assessment, questions should be asked as to what constitutes valid patient feedback from both a professional and patient perspective. If the acceptability and feasibility of patient feedback tools continue to be judged on their internal structure alone, we are at risk of developing tools that ignore other influential variables such as the inclusion of narrative comments and subjective experiences capable of facilitating learner engagement and reflection. ${ }^{50}$ Is 'valid' patient feedback a psychometrically sound tool that can be 'gamed' by both patients and physicians, ${ }^{51}$ or is a broader understanding of validity that relates to its ability to encourage actionable and beneficial change regardless of its source, format or design now required? Experience is valid for the person involved. As stated by Downing et al. validity is comprised of five 
domains including internal structure i.e. psychometric validation, response process i.e. how do participants involved respond to the assessment? And consequence, i.e. what are the intended and unintended consequences of the intervention/assessment? ${ }^{48}$ Typically, those involved in the design, administration and evaluation of patient feedback tool have focused on its internal structure only. Emerging evidence suggests some patients alter their response scores due to anticipated fear of reprisals. ${ }^{52}$ Once one validity domain is undermined, so is that tool's ability to be used as an equitable summative assessment tool. This reiterates the need to clarify the intended purpose of patient feedback i.e. formative or summative. Why are we collecting patient feedback? What do we intend to do with it? If we are trying to achieve enhanced reflection, quality improvement and professional development through the exploration of patient experience, then traditional notions of validity (i.e. psychometric validation only), arguably become redundant. As a result, in contrast to statements such as 'high quality feedback will only be achieved if the instruments have good psychometric properties, ${ }^{53}$ the authors of this review conclude it is the ability of patient feedback tools to encourage reflective practice and actionable change that have demonstrable PPI throughout their development that now require more attention, support and focus.

Thirdly the existing culture of organisations and professionals arguably restricts patient involvement and subsequent acceptance. Cultures across the education continuum favour objective and behavioural criteria over subjective experience. Exclusion, or rejection, of feedback can lead to patient experience being medicalised. This situation may be further exacerbated by application of a professional framework that is perhaps currently misaligned with patient perspectives and desires.$^{54}$

\section{Implications}

Although primarily focused on psychiatry, this review has clear practical and policy related implications for other healthcare disciplines and services more generally. Firstly, patient feedback tools should be co-produced from the outset to ensure patient and professional desires are acknowledged. Secondly, the intended purpose and scope, i.e. patient experience, satisfaction or expectation must be clearly articulated prior to the design and evaluation of any patient feedback tool. Thirdly, patient participation, psychometric validation, or absence of missing data should not be used to determine patient acceptability 
alone, and finally, those working in healthcare services should reconsider what constitutes valid patient feedback for both patients and physicians. Ultimately, what is it we are trying to achieve when asking for patient feedback? If it is to enhance patient experience, patient safety and quality of care then we must ask what matters most to patients to ensure services and individuals are delivering this.

\section{Strengths and limitations}

Strengths of this research include its rigorous application of accepted guidelines, 2021 collaboration with a patient research partner and inclusion of grey literature. However, its limitations must also be acknowledged. Most of the evidence reviewed relied primarily on volunteer samples. ${ }^{40}$ Results may not therefore be representative of the wider population. In some cases, doctors also chose their own patients, introducing possible patient selection and response bias. This review also only included articles published in the English language. Potential publication bias is therefore acknowledged. Finally, included articles rarely described the level or detail of PPI initiatives. The review is therefore reliant on available information at the time of publication.

\section{Conclusion}

Whilst inherently agreed that patient feedback tools should incorporate PPI throughout their development, current methods rely primarily on the professional perspective only. Existing tools are therefore undermined by a number of identified flaws, assumptions and contradictions including the belief that: professional and patient agendas are synonymous; psychometric validation is indicative of patient acceptability; and psychiatric patients do not have the capacity or desire to be involved. A reconsideration of what constitutes valid patient feedback is required. Future patient feedback tools should also be co-produced from the outset to ensure they are valued by all those involved.

\section{References}

1. Sheard L, Marsh C, O'Hara J, et al. The Patient Feedback Response Framework - Understanding why UK hospital staff find it difficult to make improvements based on patient feedback: A qualitative study. Social Science \& Medicine 2017;178:19-27. doi: http://dx.doi.org/10.1016/i.socscimed.2017.02.005

2. Reinders $M E$, Ryan $B L$, Blankenstein $A H$, et al. The effect of patient feedback on physicians' consultation skills: a systematic review. Academic Medicine 2011;86(11):1426-36.

3. Bodur S, Filiz E. A survey on patient safety culture in primary healthcare services in Turkey2009. 
4. Altmiller $\mathrm{G}$. The role of constructive feedback in patient safety and continuous quality improvement. The Nursing Clinics Of North America 2012;47(3):365-74. doi: 10.1016/j.cnur.2012.05.002

5. Sir Keith Pearson. Taking revalidation forward: improving the process of relicensing for doctors, 2017:80.

6. Archer J, Cameron N, Laugharn K, et al. Interim Report 2016 [Available from: http://www.gmcuk.org/UMbRELLA interim report FINAL.pdf 65723741.pdf.

7. Royal College of Psychiatrists. Supporting information for appraisal and revalidation: guidance for psychiatrists 2014 [Available from: http://www.rcpsych.ac.uk/usefulresources/publications/collegereports/cr/cr194.aspx.

8. Barbato A, Bajoni A, Rapisarda F, et al. Quality assessment of mental health care by people with severe mental disorders: A participatory research project. Community mental health journal 2014;50(4):402-08.

9. Delaney KR, Johnson ME, Fogg L. Development and testing of the combined assessment of psychiatric environments: a patient-centered quality measure for inpatient psychiatric treatment. Journal Of The American Psychiatric Nurses Association 2015;21(2):134-47. doi: $10.1177 / 1078390315581338$

10. Bjertnaes O, Iversen $\mathrm{HH}$, Kjollesdal J. PIPEQ-OS--an instrument for on-site measurements of the experiences of inpatients at psychiatric institutions. BMC Psychiatry 2015;15:234-34. doi: 10.1186/s12888-015-0621-8

11. Campbell J, Narayanan A, Burford B, et al. Validation of a multi-source feedback tool for use in general practice. Education for Primary Care 2010;21(3):165-79.

12. Campbell J, Wright C. GMC Multi-Source Feedback Questionnaires. Interpreting and handling multisource feedback results: Guidance for appraisers, 2012.

13. Tait L, Lester H. Encouraging user involvement in mental health services. Advances in psychiatric treatment 2005;11(3):168-75.

14. Goodwin I. A qualitative analysis of the views of in-patient mental health service users. Journal of Mental Health 1999;8(1):43-54.

15. Gayet-Ageron A, Agoritsas T, Schiesari L, et al. Barriers to Participation in a Patient Satisfaction Survey: Who Are We Missing? PLOS ONE 2011;6(10) doi: 10.1371/journal.pone.0026852

16. Crawford MJ, Rutter D, Manley $C$, et al. Systematic review of involving patients in the planning and development of health care. Bmj 2002;325(7375):1263.

17. Armstrong $\mathrm{N}$, Herbert $\mathrm{G}$, Aveling EL, et al. Optimizing patient involvement in quality improvement. Health Expect 2013;16(3):e36-47. doi: 10.1111/hex.12039

18. O'Regan C, Ryan M. Patient satisfaction with an emergency department psychiatric service. International Journal Of Health Care Quality Assurance 2009;22(5):525-34.

19. Thornicroft G, Brohan E, Kassam A, et al. Reducing stigma and discrimination: Candidate interventions. International Journal of Mental Health Systems 2008;2(1):3.

20. Moher D, Liberati A, Tetzlaff J, et al. Preferred reporting items for systematic reviews and metaanalyses: the PRISMA statement. Annals of internal medicine 2009;151(4):264-69.

21. Khan KS, Ter Riet G, Glanville J, et al. Undertaking systematic reviews of research on effectiveness: CRD's guidance for carrying out or commissioning reviews: NHS Centre for Reviews and Dissemination 2001.

22. Baines RL, Regan de Bere S. Optimizing patient and public involvement (PPI): Identifying its "essential" and "desirable" principles using a systematic review and modified Delphi methodology. Health Expectations 2017 doi: 10.1111/hex.12618

23. Sampson M, McGowan J, Cogo E, et al. An evidence-based practice guideline for the peer review of electronic search strategies. Journal of clinical epidemiology 2009;62(9):944-52.

24. Fourth International Conference on Grey Literature: New Frontiers in Grey Literature. ; 1999 4-5 October; Washington D.C. USA. Grey Literature Network Service. 
25. Mourad Ouzzani, Hossam Hammady, Zbys Fedorowicz, et al. Rayyan - a web and mobile app for systematic reviews 2016 [Available from: https://rayyan.qcri.org/ accessed 30.11.2016.

26. Gysels MH, Evans C, Higginson IJ. Patient, caregiver, health professional and researcher views and experiences of participating in research at the end of life: a critical interpretive synthesis of the literature. BMC Medical Research Methodology 2012;12(1):1-17. doi: 10.1186/14712288-12-123

27. Dixon-Woods M, Cavers D, Agarwal S, et al. Conducting a critical interpretive synthesis of the literature on access to healthcare by vulnerable groups. BMC Medical Research Methodology 2006;6(1):1-13. doi: 10.1186/1471-2288-6-35

28. NVivo 11. NVivo qualitative data analysis Software: QSR International Pty Ltd, 2012.

29. Glaser B. Discovery of grounded theory: Strategies for qualitative research: Routledge 2017.

30. Flemming K. Synthesis of quantitative and qualitative research: an example using Critical Interpretive Synthesis. Journal of Advanced Nursing 2010;66(1):201-17. doi: 10.1111/j.13652648.2009.05173.x

31. Buckley S, Coleman J, Davison I, et al. The educational effects of portfolios on undergraduate student learning: a Best Evidence Medical Education (BEME) systematic review. BEME Guide No. 11. Med Teach 2009;31(4):282-98. doi: 10.1080/01421590902889897 [published Online First: 2009/05/01]

32. Tai J, Molloy E, Haines T, et al. Same-level peer-assisted learning in medical clinical placements: a narrative systematic review. Medical Education 2016;50(4):469-84. doi: $10.1111 /$ medu.12898

33. Thomas J, Harden A. Methods for the thematic synthesis of qualitative research in systematic reviews. BMC Medical Research Methodology 2008;8(1):1-10. doi: 10.1186/1471-2288-8-45

34. Royal College of Psychiatrists. Revalidation guidance for psychiatrists, 2011:1-56.

35. Academy of Medical Royal Colleges. Multisource Feedback, Patient Surveys and Revalidation: report and recommendations from the MSF Work Group of the Academy of Medical Royal Colleges 1-14.

36. Royal College of Psychiatrists. Why choose ACP 360? 2017 [Available from: http://www.rcpsych.ac.uk/workinpsychiatry/qualityimprovement/acp360/whychooseacp36 0. aspx accessed 07.07.2016.

37. Violato $\mathrm{C}$, Lockyer JM, Fidler H. Assessment of psychiatrists in practice through multisource feedback. Canadian Journal Of Psychiatry Revue Canadienne De Psychiatrie 2008;53(8):52533.

38. Mason R, Power S, Parker-Swift J, et al. 360-degree appraisal: a simple pragmatic solution. Clinical Governance: An International Journal 2009;14(4):295-300. doi: 10.1108/14777270911007818

39. Stewart RF, Kroth PJ, Schuyler M, et al. Do electronic health records affect the patientpsychiatrist relationship? A before \& after study of psychiatric outpatients. BMC Psychiatry 2010;10:3-3. doi: 10.1186/1471-244X-10-3

40. Campbell JL, Richards SH, Dickens A, et al. Assessing the professional performance of UK doctors: an evaluation of the utility of the General Medical Council patient and colleague questionnaires. Quality \& Safety In Health Care 2008;17(3):187-93. doi: 10.1136/qshc.2007.024679

41. Campbell JL, Roberts $M$, Wright $C$, et al. Factors associated with variability in the assessment of UK doctors' professionalism: analysis of survey results. BMJ (Clinical Research Ed) 2011;343:d6212-d12. doi: 10.1136/bmj.d6212

42. Lelliott $P$, Williams $R$, Mears A, et al. Questionnaires for 360 -degree assessment of consultant psychiatrists: Development and psychometric properties. The British Journal of Psychiatry 2008;193(2):156-60. doi: http://dx.doi.org/10.1192/bjp.bp.107.041681 
43. Laugharne R, Pant M. Sector and functional models of consultant care: In-patient satisfaction with psychiatrists. The Psychiatrist 2012;36(7):254-56. doi: http://dx.doi.org/10.1192/pb.bp.111.037333

44. Schröder A, Wilde Larsson B, Ahlström G. Quality in psychiatric care: an instrument evaluating patients' expectations and experiences. International journal of health care quality assurance 2007;20(2):141-60.

45. Zendjidjian XY, Auquier $P$, Lancon $C$, et al. The SATISPSY-22: Development and validation of a French hospitalized patients' satisfaction questionnaire in psychiatry. European Psychiatry 2015;30(1):172-78.

46. Laugharne R, Priebe S. Trust, choice and power in mental health. Soc Psychiatry Psychiatr Epidemiol 2006;41(11):843-52. doi: 10.1007/s00127-006-0123-6

47. Boyer L, Lançon C, Baumstarck K, et al. Evaluating the impact of a quality of life assessment with feedback to clinicians in patients with schizophrenia: randomised controlled trial. The British Journal Of Psychiatry: The Journal Of Mental Science 2013;202:447-53. doi: 10.1192/bjp.bp.112.123463

48. Downing SM. Validity: on the meaningful interpretation of assessment data. Medical education 2003;37(9):830-37.

49. Gill SD, Dolley PJ, Dunning TL, et al. Evaluating health services with point of service feedback: perspectives and experiences of patients, staff and community volunteers in an inpatient rehabilitation facility. Disability And Rehabilitation 2015;37(21):1997-2005.

50. Overeem K, Lombarts MJMH, Arah OA, et al. Three methods of multi-source feedback compared: A plea for narrative comments and coworkers' perspectives'. Informa healthcare 2010;32(2):141-47.

51. Rozenblum R, Bates DW. Patient-centred healthcare, social media and the internet: the perfect storm?: BMJ Publishing Group Ltd, 2013.

52. Edwards $A$, Evans $R$, White $P$, et al. Experiencing patient-experience surveys: a qualitative study of the accounts of GPs. The British Journal of General Practice 2011;61(585):e157-e66. doi: 10.3399/bjgp11X567072

53. Royal College of Psychiatrists. What is ACP 360? 2017 [Available from: http://www.rcpsych.ac.uk/workinpsychiatry/qualityimprovement/acp360/whatisACP360.as px accessed 07.07.2017.

54. Jacob KS. Patient experience and psychiatric discourse. Psychiatrist 2012;36(11):414-17. 